(1) $P$ Observatori de

Bioètica i Dret

ISSN: $1886-5887$

\section{Revista de Bioética y Derecho}

Publicación del Máster en Bioética y Derecho nww.bioeticayderecho.ub.es

ARTíCUlo

\title{
La investigación internacional en seres humanos: de la justicia distributiva a la justicia social
}

\author{
Ricardo PÁEZ MoRENo*
}

Índice

Introducción

1. La investigación internacional patrocinada por la Industria Farmacéutica en los países de mediano o bajo ingreso

2. La bioética de la investigación biomédica internacional en seres humanos

3. La justicia social según Powers y Faden

4. Aportes desde la justicia social al debate bioético

* Ricardo Páez Moreno. Doctor en Ciencias. Profesor del Programa de Maestría y Doctorado, Universidad Nacional Autónoma de México. ricardomsps@yahoo.com 


\title{
Resumen
}

La investigación internacional en seres humanos patrocinada por la industria farmacéutica ha sido objeto de múltiples pronunciamientos bioéticos. Sin embargo ha hecho falta una reflexión desde la justicia social, que tome en cuenta los determinantes sociales de la salud en cada lugar. En este artículo se ofrecen algunos aportes para la bioética de la investigación internacional en seres humanos a partir de la justicia social.

Palabras clave: investigación internacional en seres humanos; ética; bioética; justicia social; industria farmacéutica; determinantes sociales de la salud.

\begin{abstract}
Many bioethical papers have been written about the international research in humans sponsored by the pharmaceutical industry. The perspective of social justice, which takes into account the social determinants of health in each place, however, has been lacking in most of these papers. This article offers some contributions to the bioethics of international human-subjects research based on social justice.
\end{abstract}

Key words: international human-subjects research; ethics; bioethics; social justice; pharmaceutical industry; social determinants of health. 


\section{Introducción}

La Industria farmacéutica (IF) ha desarrollado desde fines del siglo pasado una estrategia multinacional para llevar a cabo su investigación y desarrollo (I\&D), consistente en realizar protocolos de investigación sobre todo fase $\mathrm{III}^{1}$ en múltiples países simultáneamente, en los cuales puede obtener pacientes e instalaciones con mayor facilidad que en los lugares de origen donde las investigaciones son diseñadas, además de ahorrarse costos y tener menos restricciones legales. Para esto hace uso de las Contract Research Organizations (CRO) que son empresas lucrativas dedicadas a rastrear lugares dónde realizar las investigaciones, reclutar investigadores y pacientes, e incluso hasta diseñar los protocolos de investigación. ${ }^{2}$ El beneficio obtenido por la IF en términos económicos es cuantioso sobre todo cuando se obtiene la patente del producto exitoso y logra comercializarse, comparado con el beneficio aportado a los pacientes e instituciones huéspedes, lo cual puede ser calificado como explotación. ${ }^{3}$

Varios documentos internacionales ofrecen un buen marco de referencia para evitar la explotación. ${ }^{4}$ Los diversos aportes han sido clasificados bajo el esquema de "disponibilidad

\footnotetext{
${ }^{1}$ Los ensayos clínicos tienen cuatro fases (I-IV). La fase I, que se hace en humanos, es para comprobar la toxicidad del producto investigado. La fase II es aquella en la que el fármaco debe compararse con los mejores medicamentos disponibles para el tratamiento de la enfermedad implicada, y si tales fármacos no existen la comparación sería con placebo. La finalidad de ésta fase es la de establecer mediciones preliminares de la relación eficacia terapéutica/toxicidad, así como definir la dosis óptima o sus límites de variación en la condición a tratar. En la fase III, aparte de verificar la eficacia del fármaco, se busca determinar manifestaciones de toxicidad previamente no detectadas, y se obtiene una mejor perspectiva de la relación entre seguridad y eficacia, parámetros que han de cuantificarse en el contexto del desorden que se pretenda tratar. Esta es la fase más propia de las investigaciones internacionales, puesto que requiere gran cantidad de pacientes, investigadores y centros hospitalarios. La fase IV consiste en el seguimiento del fármaco después de que ha sido comercializado, y se busca básicamente la detección de toxicidad previamente insospechada, así como la evaluación de la eficacia a largo plazo.
}

2 PETRYNA, ADRIANA, When Experiments Travel. Clinical Trials and the Global Search for Human Subjects, Princeton University Press, NJ-Oxford, 2009, p. 11-13; GLICKMAN, SETH W., et.al. "Ethical and Scientific Implications of the Globalization of Clinical Research", N Engl J Med 360, Num 8, (2009), 816-823; ANGELL, MARCIA, The Truth About the Drug Companies. How They Deceive Us and What To Do About It, Random House, NY, 2004, p. 27-31 y 89-106.

${ }^{3}$ PÁEZ, RICARDO - GARCIA DE ALBA, JAVIER, "International Research and J ust Sharing of Benefits in Mexico", Developing World Bioeth, Vol 9, Num 2, (2009), 65-73.

${ }^{4}$ THE NATIONAL COMMISSION FOR THE PROTECTION OF BIOMEDICAL AND BEHAVIORAL RESEARCH, "The Belmont Report" part 3, Justice, Ethical Principles and Guidelines for the protection of human subjects of research, NIH, Bethesda, Maryland, 1979, en: http://ohsr.od.nih.gov/guidelines/belmont.html\#gob3, [Accesado: 9 de junio de 2011]; WORLD MEDICAL ASSOCIATION, "Declaration of Helsinki" Paragraphs 32 and 33, WMA, Ferney-Voltaire, France, October 2008, en: http://www.wma.net/en/30publications/10policies/b3/, [Accesado: 9 de junio de 2011]; COUNCIL FOR INTERNATIONAL ORGANIZATIONS OF MEDICAL SCIENCES, "International Ethical Guidelines for Biomedical Research Involving Human Subjects", CIOMS, Geneva 2002, en: http://www.cioms.ch/publications/layout_guide2002.pdf, [Accesado: 9 de junio de 2011]; NUFFIELD COUNCIL OF BIOETHICS. "The Ethics of Research Related to Care in Developing Countries", NCB, London, 2002, en: http://www. nuffieldbioethics.org/sites/default/files/Ethics\% 20 of\% 20research\% 20related\% 20to\% 20healthcare 
razonable" que busca que el producto exitoso sea disponible en la comunidad huésped al terminar el estudio, y el de "justos beneficios" que se refiere a la justa distribución de beneficios que les corresponde recibir a los participantes, a la comunidad y a las instituciones médicas huéspedes. ${ }^{5}$

Pero más importante aún para la labor bioética ha sido la. toma de conciencia de los factores estructurales condicionantes de graves injusticias imperantes en la investigación biomédica y fuera de ella, también llamados Determinantes Sociales de la Salud (DSS), y que son aquellos factores ambientales, sociales, relacionales, más allá de los biológicos, que ocasionan que una persona o comunidad se enfermen. ${ }^{6}$ El contemplar a los individuos como seres sociales cuyas necesidades, capacidades y posibilidad de elegir han de ser contextualizadas, amplía la mirada de la bioética a una comprensión más grande de la justicia. Los contextos se vuelven un punto de partida imprescindible para una reflexión bioética sobre lo justo. Es decir, es necesario ir más allá de la justicia distributiva y adentrarse en la justicia social.

La pregunta a la que se pretende responder en este artículo es: dada la injusta realidad de algunos países en donde se realiza la investigación biomédica internacional de la IF, la cual está fuertemente condicionada por el interés financiero, ¿cuál sería el aporte que podría ofrecer al debate bioético un enfoque basado en la justicia social? Para ello se describirán en primer lugar, algunas nociones sobre la investigación internacional patrocinada por la IF; en segundo lugar, se desarrollarán dos importantes enfoques bioéticos con que se ha abordado esta realidad; en tercer lugar, se presentará el aporte desde la justicia social tal y como lo han trabajado Powers y Faden; y en último lugar, se desarrollarán algunas pautas que aporta la justicia social al debate bioético sobre la materia.

\section{La investigación internacional patrocinada por la IF en los países de mediano o bajo ingreso}

La investigación internacional es una modalidad reciente de (I\&D) patrocinada por corporativos transnacionales que cuentan entre sus negocios con grandes firmas farmacéuticas, y cuya aplicación es llevada a cabo en diversos países y centros hospitalarios del mundo. La IF es el mayor productor del arsenal terapéutico actual, y crecientemente ha globalizado y descentralizado la investigación

\%20in\%20developing\%20countries\%201.pdf, [Accesado: 9 de junio de 2011]; NATIONAL BIOETHICS ADVISORY COMMISSION, "Ethical and Policy Issues in Research Involving Human Participants Vol 1", NBAC, Bethesda, Maryland, 2001, en: http://bioethics.georgetown.edu/nbac/human/overvol1.pdf [Accesado: 9 de junio de 2011]; UNITED NATIONS EDUCATIONAL, SCIENTIFIC AND CULTURAL ORGANIZATION, "Universal Declaration on Bioethics and Human Rights, Arts. 2, 4, 15 y 24", UNESCO, Paris, 2005, en: http://portal.unesco.org/en/ev.php-URL_ID=31058\&URL_DO=DO_TOPIC\&URL_SECTION=201.html [Accesado: 9 de junio de 2011].

5 PARTICIPANTS IN THE 2001 CONFERENCE ON ETHICAL ASPECTS OF RESEARCH IN DEVELOPING COUNTRIES,, "Moral Standards for Research in Developing Countries. From 'Reasonable Availability' to 'Fair Benefits' ", Hastings Cent Rep, 34, Num 3, (2004), 17-27.

${ }^{6}$ La literatura sobre ética de la salud pública está actualmente ampliando la visión de sus debates bioéticos más allá de los asuntos relacionados con el paciente individual, para considerar también las respuestas sociales a la salud-enfermedad en las poblaciones. Ver: VENKATAPURAM, SRIDHAR, - MARMOT, MICHAEL, "Epidemiology and Social J ustice in Light of Social Determinants of Health in Research", Bioeth, 23, Num 2, (2009), 79-89. 
clínica, la cual ha estado a la alza recientemente ${ }^{7}$. De hecho la mayoría de las tareas de investigación clínica actual dependen del patrocinio de la IF. ${ }^{8}$

La investigación internacional está muy condicionada por el interés financiero de la $\mathrm{IF} .{ }^{9}$ Según Bodenheimer ${ }^{10}$ con el nuevo énfasis en la prevención y tratamiento de enfermedades crónicas, ha cambiado la investigación en medicamentos. Para establecer la eficacia y la seguridad de este tipo de medicamentos, los ensayos clínicos necesitan ser largos, duraderos y realizados en múltiples centros puesto que uno solo no puede reclutar el número suficiente de pacientes para asegurar la validez estadística. $^{11}$

Además, para acelerar los ensayos clínicos, la IF está cambiando de los centros médicos académicos a las CRO que contratan a médicos, científicos, farmaceutas, especialistas en estadística y gerentes, y les ofrecen un menú de servicios. La competencia por el dinero producto de los ensayos se ha configurado como cientos de $\mathrm{CRO}$, centros médicos académicos y sitios no académicos independientes, "luchando por la parte más grande del pastel." ${ }^{12}$

Respecto al diseño de los protocolos, tradicionalmente los investigadores ajenos a los ensayos diseñaban los protocolos de investigación, pero actualmente es la IF quien hace los ensayos y contrata a investigadores para dar su firma, sin posibilidades de modificación. Y en relación con la autoría, antes escribía un autor principal, pero actualmente se está dando el síndrome: autor no escritor - no autor escritor (non writing author - non author writer). ${ }^{13}$ Cada vez más se reporta en la literatura cómo la IF genera artículos por escritores "fantasma" y busca publicarlos en revistas científicas. ${ }^{14}$

Por parte de los países huéspedes, la oportunidad que representan las investigaciones internacionales ante la falta de tratamientos debido al insuficiente presupuesto destinado por el

\footnotetext{
${ }^{7}$ Entre 1995 y 2005, el número de países huéspedes de investigación internacional fuera de Estados Unidos (EU) creció más del doble, mientras que la proporción de investigaciones realizadas en Europa y EU disminuyó. Ver: GLICKMAN, SETH W. et.al, "Ethical and Scientific Implications..." op. cit. 818.

${ }^{8}$ PETRYNA, ADRIANA, When Experiments... op. cit. 5-9; ANGELL, MARCIA. The Truth About... op. cit. 10-13.

${ }^{9}$ PÀEZ, RICARDO, "Retos a la justicia en la investigación global de la industria farmacéutica", Cuadernos de Bioética, Vol XXI, No 3, (2010), 375-390.

${ }^{10}$ BODENHEIMER, THOMAS, "Uneasy alliance - Clinical Investigators and the Pharmaceutical Industry", N Eng J Med, 342 Num 20, (2000), 1539-1544.

${ }^{11}$ Habría que añadir que al realizar investigaciones en países de mediano o bajo ingreso, que generalmente tienen grandes poblaciones, la IF se beneficia por la facilidad para lograr el amplio reclutamiento de pacientes requerido para la aprobación de los ensayos clínicos por la Food and Drug Administration de los EU. Además, para licitar las investigaciones hay que pasar menos trámites regulatorios burocráticos. PETRYNA, ADRIANA, When Experiments... op. cit. 18 y 20.

${ }^{12}$ Para ver otras críticas a las CRO: PETRYNA, ADRIANA, "Clinical Trials Offshores: On Private Sector Science and Public Health", BioSocieties, 2, (2007), 21-40.

${ }^{13}$ BODENHEIMER, THOMAS. Uneasy alliance... op. cit. 1544.

${ }^{14}$ FUGH-BERMAN, ADRIANE J. "The Haunting of Medical Journals. How Ghostwriting sold 'HRT' ", PLos Medicine Vol 7, 9, (2010) e1000335. doi:10.1371/journal.pmed.1000335. Otras razones para realizar investigaciones internacionales son: el creciente negocio mundial de las "me-too drugs", es decir, productos desarrollados rapidamente imitando productos exitosos con mínimos cambios farmacológicos para ganar nichos atractivos de mercado, y probar en pacientes en países poco medicalizados y por lo tanto más sensibles a los productos experimentales. Ver: PETRYNA, ADRIANA. When Experiments... op. cit. 20-21 y 69-70.
} 
Estado a la salud pública, aunado a una clase médica mal remunerada, el cual es un importante DSS, hace de aquellas una oferta difícil de rechazar tanto para los pacientes como para los investigadores. ${ }^{15}$

Otro factor muy importante a tener en cuenta en muchos de los países huéspedes de investigación internacional son los DSS. Estos son una medida adoptada por la Organización Mundial de la Salud (OMS) para descubrir el impacto que las causas sociales originan sobre la salud de la población y diseñar planes de acción conjunta. La OMS publicó recientemente varios DSS desarrollados por expertos en diversas partes del mundo, entre los que incluyen: los efectos de la globalización, el desarrollo en la infancia temprana, la atención en los sistemas sanitarios, la condición de vida en las ciudades, las condiciones laborales, la equidad de género y la exclusión social. ${ }^{16}$

En Canadá y varios países, se ha adquirido bastante evidencia de que las circunstancias socioeconómicas de grupos e individuos son de igual o mayor importancia para el estado de salud de la población, que la atención médica y las conductas personales saludables, patrones de alimentación o incluso el tabaquismo. El peso de la evidencia sugiere que los DSS tienen un impacto directo en la salud de los individuos y poblaciones, y son los mejores indicadores de prevención sobre la salud individual y poblacional, condicionan la elección de estilos de vida, e interactúan con cualquier otro determinante para generar salud. En términos de salud poblacional, es bien conocido que entre mayor sea el tamaño de las disparidades entre estratos económicos y sociales de una población dada, más se afecta el estado de salud del conjunto. ${ }^{17}$

En países de mediano o pobre ingreso, o incluso países "emergentes" como México, donde por una parte existen múltiples DSS que afectan negativamente la salud y el bienestar de la población, y por otra se realiza gran cantidad de investigación internacional, es del todo relevante hacerse la pregunta bioética por lo justo para los pacientes, investigadores y comunidades huéspedes.

\section{La bioética de la investigación biomédica internacional en seres humanos}

La ética de la investigación internacional en seres humanos tiene como referente una serie de documentos internacionales que norman con el fin que la investigación se lleve a cabo de manera correcta cuando se realiza en países pobres, por el riesgo de explotar a los sujetos de investigación

\footnotetext{
${ }^{15}$ PETRYNA, ADRIANA, When experiments... op. cit. 31; PÁEZ, RICARDO - GARCIA DE ALBA, JAVIER, "International Research..." op.cit. 70-73.

${ }^{16}$ WORLD HEALTH ORGANIZATION (WHO), Commission on Social Determinants of Health, "Final reports and additional documents of the Knowledge Networks", WHO, Geneve, August 2008, en: http://www.who.int/social_determinants/thecommission/finalreport/en/index.html [Último Acceso 16 de junio de 2011].

${ }^{17}$ PUBLIC HEALTH AGENCY OF CANADA, "The Social Determinants of Health: An Overview of the Implications for Policy and the Role of the Health Sector". Ottawa, Ontario, March 2004, en: http://www.phac-aspc.gc.ca/ph-sp/oi-ar/pdf/01_overview_e.pdf [Accesado 16 de junio de 2011].
} 
debido a su situación de vulnerabilidad. Al respecto han surgido consideraciones respecto al concepto de "disponibilidad razonable" y "justicia distributiva."

El esquema llamado "disponibilidad razonable" que pretende que el producto investigado con éxito sea disponible en la comunidad huésped al finalizar el estudio, ${ }^{18}$ se ve reflejado en las normas CIOMS en su versión actual ${ }^{19}$ y pretende evitar cualquier tipo de explotación en los países pobres en donde se investiga, puesto que la disponibilidad del medicamento o vacuna probada no se podrá tener de manera regular en el país pobre, mientras que su acceso para los habitantes de los países ricos es mucho más fácil (o la gente lo paga por sí misma, o el sistema de salud pública o los seguros lo hacen). Sin embargo algunos consideran que este escenario es típico de la explotación y suscita diversas preocupaciones. ${ }^{20} \mathrm{El}$ problema principal del esquema de la disponibilidad razonable está en que garantiza qué beneficio se va a compartir, pero no especifica cuánto beneficio debe darse a las partes involucradas. ${ }^{21}$

Se han hecho algunos ajustes al beneficio razonable a partir de un esquema llamado "justa distribución de beneficios", el cual descansa en tres principios: la investigación debe tener valor social, es decir, debe tener que ver con algún problema del país huésped; los sujetos de investigación deben ser seleccionados imparcialmente, de acuerdo a los objetivos de la investigación, evitando toda justificación basada en la pobreza o vulnerabilidad; y la investigación debe tener un cociente riesgo/beneficio favorable. ${ }^{22}$

Los proponentes de este esquema quieren evitar ante todo la explotación, la cual es una falta ética muy específica que se ha confundido con otras formas de dañar a las personas. Es por ello que adoptan el concepto de explotación de A. Wertheimer. ${ }^{23}$ Una correcta comprensión de la explotación

\footnotetext{
${ }^{18}$ PARTICIPANTS IN THE 2001 CONFERENCE ON ETHICAL ASPECTS OF RESEARCH IN DEVELOPING COUNTRIES. "Moral Standards...", op. cit. 17-18.

${ }^{19}$ COUNCIL FOR INTERNATIONAL ORGANIZATION OF MEDICAL SCIENCES, "International Ethical Guidelines..." op. cit. Guideline 10.

${ }^{20}$ Se parte de la definición de explotación dada por Ruth Macklin: "ocurre explotación cuando las personas -o las agencias ricas o poderosas- obtienen ventaja de la pobreza, debilidad, o dependencia de los otros, usándolos para servir sus propias metas, sin beneficios adecuados para compensar a los individuos o a los grupos que son dependientes o menos poderosos." MACKLIN, RUTH, "Ética de la investigación internacional", Acta Bioeth, año X, No 1, (2004), 32.

${ }^{21}$ EMMANUEL, EZEQUIEL, "A World of Research Subjects. Introduction”, Hastings Cent Rep 28 Num 6, (1998), 25; CROUCH, ROBERT A. - ARRAS, JOHN D, "AZT Trials and Tribulations”, Hastings Cent Rep 28 Num 6, (1998), 26-34. Otras críticas que se han hecho a este abordaje son: la noción de beneficios es muy estrecha, sin considerar otros bienes como los sociales; el beneficio depende del éxito del medicamento en estudio si no existe un contrato previo de distribución de beneficios; no puede garantizarse un beneficio de parte de las compañías patrocinadoras o de los gobiernos; el beneficio en los protocolos fase III tarda en ser demostrado lo cual retarda mucho la distribución del mismo; la población no puede decidir por sí misma cuál es su mejor beneficio, etc. Ver: PARTICIPANTS IN THE 2001 CONFERENCE ON ETHICAL ASPECTS OF RESEARCH IN DEVELOPING COUNTRIES. Moral Standards... op. cit. 20-22

${ }^{22}$ PARTICIPANTS IN THE 2001 CONFERENCE ON ETHICAL ASPECTS OF RESEARCH IN DEVELOPINGCOUNTRIES. "Moral Standards...", op. cit. 22

${ }^{23}$ Wertheimer sostiene que la explotación es propia de interacciones a nivel micro entre las partes, en función de un acuerdo discreto o un esfuezo cooperativo. La parte A explota a la parte B si la primera recibe un inequitativo nivel de beneficios como resultado de las interacciones de B con A. WERTHEIMER, ALAN, Exploitation. Princeton University Press, New Jersey 1996, p. 3-34.. Se reduce la explotación al nivel individual, sin tomar en cuenta el contexto social o la historia, y concibe la justicia como
} 
se supone que revela dos puntos de vista que son fundamentales en el abordaje de beneficios justos: lo más importante para evitar la explotación es asegurar que las personas que soportan los riesgos y cargas de la investigación, reciban beneficios justos que sean fruto del desarrollo de la investigación o de los resultados de esta última; y que todo tipo de beneficios que podrían derivarse de la investigación deben ser considerados en la determinación de si los beneficios son justos. Se responde no a qué beneficios reciben las comunidades huéspedes, sino al "nivel" o cantidad del beneficio. Como resultado de este esquema, las comunidades huéspedes pueden contratarse con los investigadores por un amplio rango de beneficios.

Se le critica a este segundo esquema el ser minimalista, al igual que el de beneficio razonable. No toma en cuenta las teorías de la justicia en la ética de la investigación, quedándose en lo procedimental. Se basa en el respeto a los principios de no maleficencia, beneficencia y autonomía en la investigación, y a unos justos términos de cooperación entre las partes contratantes, tomando en cuenta la consulta democrática. Esta justicia, en caso de que realmente funcionara, podría calificarse como "ventaja mutua", ofreciendo ventajas a los contratantes, pero dejando las cosas como están en la realidad, silenciando las causas de la injusticia operante en el sistema. Considera y evalúa la justicia en la investigación a partir del status quo, sin cuestionarlo. Tampoco sitúa las necesidades de salud en un contexto social, económico y político más amplio. Y por último, considera las estructuras sociales como dadas, impidiendo así distinguir las causas de la injusticia. ${ }^{24}$

\section{La justicia social según Powers y Faden ${ }^{25}$}

Para comprender más a fondo qué es lo justo en una comunidad huésped, es necesario entender los fenómenos dentro de su escenario social y no aislados de su contexto. Powers y Faden argumentan que es imposible hacer progresos en la comprensión de las demandas de justicia dentro del sistema sanitario, sin estudiar la salud pública y otros DSS, y por lo tanto, situando el análisis de lo que es justo en el contexto social y político más amplio. “... La justificación de cualquiera de las principales propuestas para la distribución de recursos estará en función de su contexto y la naturaleza y el grado de las inequidades en los determinantes de la salud, así como en la sociedad en general" ${ }^{26}$.

La auténtica prueba diagnóstica para determinar lo justo es saber si la totalidad de las relaciones sociales impone límites sistémicos en el desarrollo de las dimensiones esenciales del

transacciones ideales del mercado.

${ }^{24}$ LONDON, ALEX J, "Justice and the Development approach to International Research", Hastings Cent Rep, 35, Num 1, (2005), 24-37. Para una crítica más reciente en que se comparan las condiciones para la investigación internacional con una subasta, ver: LONDON, ALEX J, "Research at the Auction Block. Problems for the Fair Benefits Approach to Inernational Research", Hastings Cent Report 40, Num 4, (2010), 34-45. Existen varios otros aportes relacionados con el de London. En el ámbito latinoamericano: NOVOA, EDGAR, "Desafíos Bioéticos de la Cuestión Social Hoy", Revista Colombiana de Bioética Vol 2, No 2, (2007), 143-172; GARRAFA, VOLNEI, "Etica y Política”, en: TEALDI, CARLOS (Ed.) Diccionario Latinoamericano de Bioética. UNESCO, Universidad Nacional de Colombia, Bogotá, 2008, 531-533.

${ }^{25}$ POWERS, MADISON - FADEN, RUTH, Social Justice. The moral foundations of public health and health policy. Oxford University Press, NY, 2006.

${ }^{26}$ POWERS M - FADEN R. Social Justice...op. cit. Preface xi. La traducción es mía. 
bienestar, y si lo hace de manera que garantice efectos adversos profundos y penetrantes en todos los aspectos del bienestar. Sólo cuando se considera lo que la suficiencia de bienestar requiere en el mundo real, en circunstancias sociales concretas, se puede afirmar qué inequidades son más urgentes desde el punto de vista de la justicia. ${ }^{27}$

Los autores construyen su descripción de justicia social alrededor de seis dimensiones distintas del bienestar, cada una de las cuales merece atención separada dentro de su teoría de la justicia. No se trata de considerar todas las dimensiones del bienestar, sino aquellas que son de especial urgencia e importancia moral, independientemente de los planes de vida y objetivos que cada uno tenga. Tampoco se trata de definir un umbral en cada dimensión debajo del cual no puede haber una vida decente, puesto que muchos no alcanzan dicho umbral sin que por ello necesariamente ésta les falte. Consiste más bien en que si una vida humana está muy deficiente en una o más de las dimensiones, es probable que esa persona no esté experimentando un nivel suficiente de bienestar.

La suficiencia del bienestar es alcanzable sólo cuando el conjunto de dimensiones indicadoras del bienestar son satisfechas: salud, seguridad, razonamiento, respeto, vínculo y autodeterminación. Para ello, es necesario que los DSS estén en equilibrio y contribuyan a la satisfacción de estas últimas. Powers y Faden ofrecen una guía práctica para responder qué inequidades importan más cuando las condiciones de trasfondo que deberían promover la justicia, no están en su lugar. La preocupación por aquellas inequidades más urgentes es debido al hecho que las desigualdades en el mundo real están interrelacionadas, y su efecto cumulativo en el bienestar humano dependerá de su interacción causal.

La pregunta, ¿qué inequidades importan más? dependerá de cómo las instituciones actuales y las prácticas sociales estén ordenadas, y su impacto en personas y grupos diferencialmente situados. Los hechos del contexto son una información esencial, necesaria para entender qué desigualdades de cualquier tipo merecen una cuidadosa evaluación moral. Powers y Faden señalan cuatro escenarios en que los DSS pueden influir en los indicadores del bienestar:

1. Un DSS con efectos profundos y penetrantes en una dimensión del bienestar.

2. Coincidentes DSS con efectos profundos y penetrantes en una dimensión del bienestar.

3. Un DSS con efectos profundos y penetrantes en un grupo de dimensiones del bienestar.

4. Coincidentes DSS con efectos profundos y penetrantes en un grupo de dimensiones del bienestar $^{28}$.

En algunos lugares el primer escenario es el más característico, y aquí la reflexión sobre lo que requiere la justicia puede proceder sin preocuparse del impacto de cualquier otro aspecto de la estructura social y sin tener que ver con ningún otro efecto en otras dimensiones del bienestar, p.e. la distribución de recursos sanitarios escasos cuando la estructura social funciona bien. El segundo escenario requiere una más cuidadosa evaluación moral al implicar la suma de DSS que impactan en

\footnotetext{
${ }^{27}$ POWERS M - FADEN R. Social Justice...op. cit. 4-9

${ }^{28}$ POWERS M - FADEN R. Social Justice...op. cit. 65
} 
una dimensión particular del bienestar, llevando a algunas personas a estar debajo de la suficiencia del bienestar, y originando una constelación de desigualdades que sistemáticamente magnifican y refuerzan los efectos adversos iniciales. En este caso no sólo el ámbito sanitario está deficiente, sino otros DSS como la contaminación, contribuyen a la merma en la salud.

El tercer escenario se refiere a un DSS que afecta de manera profunda y penetrante varias dimensiones del bienestar, y se basa en la teoría de los bienes dominantes de M. Waltzer. ${ }^{29}$ Es el caso de un sistema de salud que no sólo impacta en su falta de cobertura, sino que también genera desigualdad, exclusión y otras formas de injusticia que se multiplican y refuerzan entre sí. Su efecto sobre las dimensiones del bienestar pude ser de manera aditiva, haciendo que quienes carecen de una dimensión del bienestar caigan bajo muchas otras, o interactivo o en cascada, llevando a muchas personas a estar bajo la suficiencia del bienestar. Lo más importante aquí es la falta de límites a los núcleos de poder (político o económico) que al acaparar bienes sociales básicos como la riqueza, provocan que grandes mayorías vivan bajo la suficiencia del bienestar.

El cuarto escenario es el más problemático es decir, coincidentes DSS con profundos y penetrantes efectos en grupos de dimensiones del bienestar. Las desigualdades traen más desigualdades, y las inequidades existentes -en los DSS y ultimadamente en las dimensiones esenciales del bienestar en sí mismas- mezclan, sostienen y reproducen una multitud de carencias de bienestar, llevando a muchas personas bajo el nivel de suficiencia en más de una dimensión. Tomadas en su conjunto, los efectos interactivos de múltiples fuentes de desventaja se añaden al muy desigual bienestar, dejando a muchos con muy pocas oportunidades en aspectos relevantes de sus vidas. En tanto las desigualdades del bienestar se multiplican, estas se refuerzan unas a otras. Estos casos son multicausales en su origen y multidimensionales en sus efectos, y coinciden con la concentración del poder político, económico, de raza o género en pocas manos. Cada esfera de deficiencia del bienestar se vuelve más difícil de revertir, los efectos desventajosos más difíciles de superar, y el objetivo de suficiencia para todos en todas las dimensiones del bienestar aún más duro de conseguir. Lograr la suficiencia es entonces simplemente incompatible con patrones densamente entretejidos de desventajas sistemáticas de este tipo. ${ }^{30}$

\section{Aportes desde la justicia social al debate bioético}

Una primer aportación que ofrece un abordaje desde la justicia social como el de Powers y Faden para el debate bioético, es que no se deben aislar los hechos del contexto social que los produce o al que éstos afectan, ya que unos y otros están profundamente interrelacionados. De otra manera se corre el riesgo de hacer una bioética ideal, distante a los reales desequilibrios estructurales existentes, la cual normalmente refuerza el status quo vigente. Para el caso de las investigaciones internacionales, es necesario tener en cuenta no sólo el ámbito sanitario y de

\footnotetext{
${ }^{29}$ Dicha teoría dice que de las desigualdades en bienes dominantes proceden mayores desigualdades en aquellas cosas que son los determinantes sociales primarios de varias dimensiones del bienestar, y el resultado de esta cadena causal es un patrón de insuficiencias sistemáticas en múltiples dimensiones del bienestar. WALTZER M, Spheres of Justice. New York: Basic Books, citado en: POWERS M - FADEN R. Social Justice...op. cit. 66-70.

${ }^{30}$ POWERS M - FADEN R. Social Justice...op. cit. 71-72
} 
investigación en salud, sino también los DSS que condicionan en buena medida las necesidades en salud y el bienestar de la población huésped.

Un enfoque bioético desde la justicia social pide comprender la estructura social del lugar donde se realizan las investigaciones, de qué manera está distribuido el poder y cómo esto afecta a la salud y al bienestar de las mayorías. En las investigaciones internacionales, es patente el gran contraste existente entre la dinámica lucrativa de la IF, y la exclusión imperante en varios países emergentes o de bajo poder adquisitivo donde se realizan las investigaciones, que de no tomarse en cuenta, se puede reforzar, p.e. al investigar en "me-too drugs" o al poner a los productos exitosos precios inalcanzables para las mayorías.

También es necesario conocer la dinámica local de la atención de la salud. En el caso de las instituciones y equipos de investigadores con carencias de recursos, suele privilegiarse la investigación internacional para atraer recursos e investigación, descuidando investigar en prioridades locales, p.e. las grandes necesidades en salud de las mayorías. Otro tanto podría decirse del uso de instalaciones y personal para los protocolos internacionales, postergando la atención de la salud de aquellos para quienes están primariamente destinados.

Un abordaje contextual pide entonces no partir de una serie de principios de justicia y aplicarlos a la realidad, sino por el contrario, describir qué pasa en la realidad, que relaciones concretas existen, qué opresiones se generan, que desigualdades importan más, para desde ahí empezar a diseñar estrategias realistas de cambio.

Detrás de crudos escenarios como el tercero o el cuarto, hay una serie de agentes cuya responsabilidad hay que detectar respecto a su deber de velar por lo justo. Entre ellos están en primer lugar las autoridades del Estado al servicio del cuidado de la salud, que es el primer agente de justicia en la modernidad. Pero también correspondería investigar la responsabilidad de quienes trabajan en la I\&D internacional de la IF, en las CRO, y de quienes negocian y aceptan investigaciones que reprueban estándares de justicia en las instituciones de salud locales, así como de los investigadores locales que aceptan colaborar en éstas. Por último, no se puede negar la responsabilidad de la sociedad civil, aunque en mucho menor grado en el caso de las mayorías empobrecidas que dan su consentimiento por necesidad.

Las reflexiones anteriores deberían llevar a una toma conjunta de conciencia de este tipo de problemas globales reflejados en lo local, y generar acciones desde distintos actores y niveles en vistas a aprovechar la investigación biomédica internacional en seres humanos para mejorar la atención de la salud y la investigación en salud local, evitando favorecer la dinámica de desigualdad imperante. 